\title{
Pink-beam serial femtosecond crystallography for accurate structure factor determination at an X-ray free electron laser
}

\author{
Karol Nass ${ }^{1}$, Camila Bacellar ${ }^{1}$, Claudio Cirelli ${ }^{1}$, Florian Dworkowski ${ }^{1}$, Yaroslav Gevorkov ${ }^{2}$, Daniel James ${ }^{1}$, Philip J. \\ M. Johnson ${ }^{1}$, Demet Kekilli ${ }^{1}$, Gregor Knopp ${ }^{1}$, Isabelle Martiel ${ }^{1}$, Dmitry Ozerov ${ }^{1}$, Alexandra Tolstikova ${ }^{2}$, Laura Vera ${ }^{1}$, \\ Tobias Weinert ${ }^{1}$, Oleksandr Yefanov ${ }^{2}$, Jörg Standfuss ${ }^{1}$, Sven Reiche ${ }^{1}$ and Christopher J. Milne ${ }^{1}$ \\ ${ }^{1}$ Paul Scherrer Institut, Forschungstrasse 111, Villigen, 5232, Switzerland \\ ${ }^{2}$ Center for Free-Electron Laser Science, Notkestrasse 85, Hamburg, 22607, Germany
}

karol.nass@gmail.com,christopher.milne@xfel.eu

Serial femtosecond crystallography (SFX) at X-ray free-electron lasers (XFELs) enables essentially radiation damage-free macromolecular structure determination using microcrystals that are too small for synchrotron studies [1]. However, SFX experiments often require large amounts of sample in order to collect highly redundant data where some of the many stochastic errors can be averaged out and accurate structure factor amplitudes determined [2]. Recently, an improvement in native-SAD phasing of SFX data was demonstrated by utilizing longer wavelengths that increased the strength of the anomalous signal [3]. This reduced up to 10-fold the number of indexed images needed for successful de novo structure determination. Another approach to reduce the number of indexed images, applicable not only to de novo phasing but also to molecular replacement strategies, is to use polychromatic (pink) Xray pulses for SFX. Theoretically, faster convergence rates of the Monte Carlo approach can be achieved by increasing the bandwidth or divergence of the $\mathrm{X}$-ray pulses $[4,5]$.

We used the capability of the Swiss free-electron laser (SwissFEL) to generate large-bandwidth X-ray pulses $(\Delta \lambda / \lambda=2.2 \%$ FWHM) and applied them in SFX with the aim of improving the partiality of Bragg spots and thus decreasing sample consumption while maintaining the data quality. Sensitive data-quality indicators such as anomalous signal from native thaumatin micro-crystals and de novo phasing results were used to quantify the benefits of using pink X-ray pulses to obtain accurate structure factor amplitudes. Compared to data measured using the same setup but X-ray pulses with typical, quasi-monochromatic XFEL bandwidth $(\Delta \lambda / \lambda=0.17$ $\%$ FWHM), up to four fold reduction in the number of indexed diffraction patterns required to obtain similar data quality was achieved. This novel approach, pink-beam SFX, facilitates the yet underutilized de novo structure determination of challenging proteins at XFELs, thereby opening the door to more scientific break-troughs.

[1] Nass, K. (2019). Acta Cryst. D 75, 211-218.

[2] Nass, K., Meinhart, A., Barends, T. R., Foucar, L., Gorel, A., Aquila, A., Botha, S., Doak, R. B., Koglin, J., Liang, M., Shoeman, R. L., Williams, G., Boutet, S. \& Schlichting, I. (2016). IUCrJ 3, 180-191.

[3] Nass, K., Cheng, R., Vera, L., Mozzanica, A., Redford, S., Ozerov, D., Basu, S., James, D., Knopp, G., Cirelli, C., Martiel, I., Casadei, C., Weinert, T., Nogly, P., Skopintsev, P., Usov, I., Leonarski, F., Geng, T., Rappas, M., Doré, A. S., Cooke, R., Nasrollahi Shirazi, S., Dworkowski, F., Sharpe, M., Olieric, N., Bacellar, C., Bohinc, R., Steinmetz, M. O., Schertler, G., Abela, R., Patthey, L., Schmitt, B., Hennig, M., Standfuss, J., Wang, M. \& Milne, C. J. (2020). IUCrJ 7, 965-975.

[4] Dejoie, C., McCusker, L. B., Baerlocher, C., Abela, R., Patterson, B. D., Kunz, M. \& Tamura, N. (2013). J. Appl. Cryst. $46,791-794$.

[5] White, T. A., Barty, A., Stellato, F., Holton, J. M., Kirian, R. A., Zatsepin, N. A. \& Chapman, H. N. (2013). Acta Cryst. D 69, $1231-1240$.

Keywords: Pink-beam; serial femtosecond crystallography; de novo protein structure determination; X-ray crystallography; SFX; SAD; single-wavelength anomalous diffraction; XFEL; large-bandwidth 